\title{
Mapping heat flow from a time series of satellite temperature images as a regional exploration tool for kimberlites
}

\author{
Neil Pendock \\ Terracore
}

\section{Introduction}

Various estimates of the Curie depth point [CDP] for countries and cratons have been made from regional and satellite magnetic surveys, the most recent in March for the whole planet using the Earth Magnetic Anomaly Grid [1]. Such estimates have applications for mineral exploration such as subaqueous hot spring gold deposits and kimberlites.

To be practically useful for exploration, spatial resolution of CDPs needs to be increased. EMAG2 has a spatial resolution of 2 arc minutes while the regional magnetic grid of the Okavango Rift Zone on a which a 3D inversion was performed to estimate CDPs [2] had a spatial resolution of $62.5 \mathrm{~m}$.
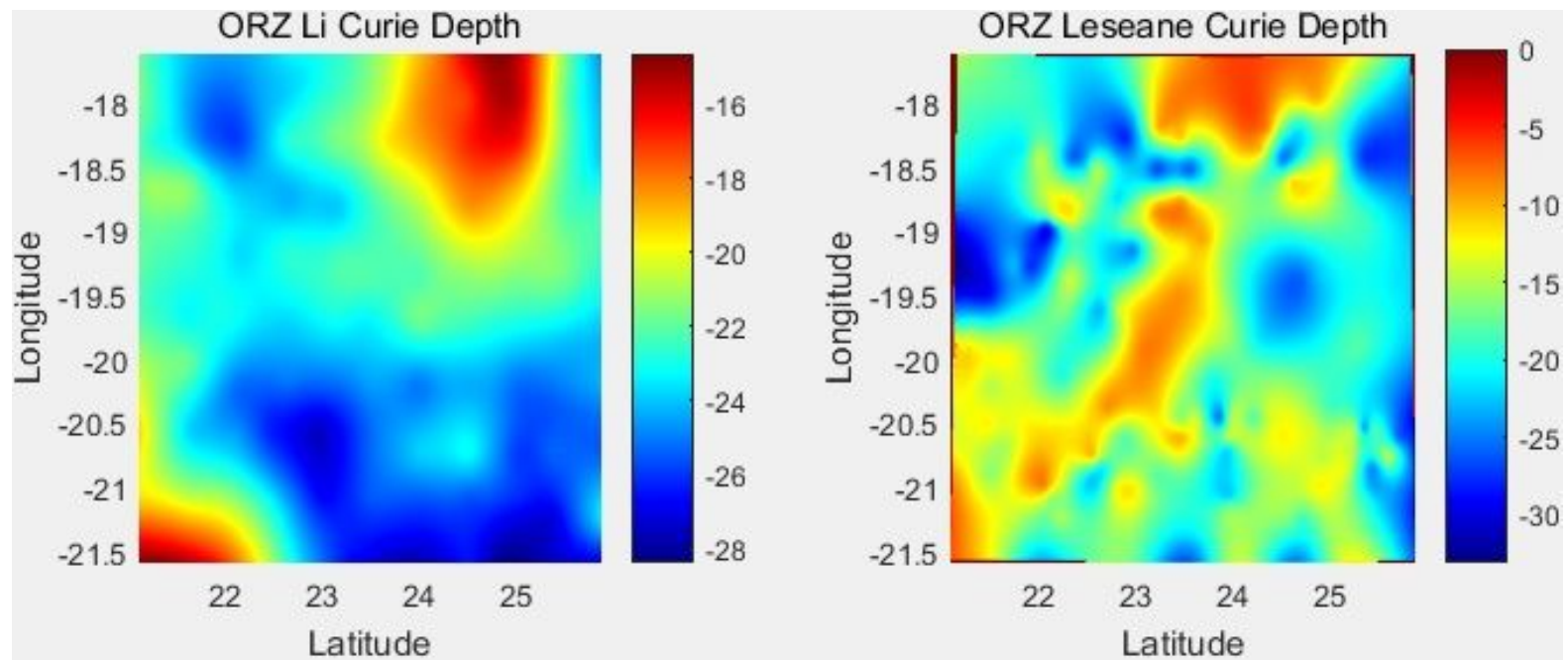

Figure 1: Curie depth estimates from satellite magnetic (left) and airborne magnetic surveys (right)

The $\sim 60 \mathrm{~km}$ wide NE-trending zone of shallow CDPs coincident with major rift-related border faults and the boundary between Proterozoic orogenic belts is lost in the EMAG2 inversion. The two CDPs have a correlation of 0.19 and shallow structure is clearly lacking in the satellite derived estimate.

High resolution regional magnetic grids are rare, at least in the public domain, while finer regional magnetic surveys raise issues for 3D inversion techniques as they are computationally demanding.

Less computationally intensive CDP estimators using the slope of the radially averaged power spectrum of the Fourier Transform of the magnetic field have issues with regards to which frequencies to use to compute the slope. The EMAG2 result, a power spectrum estimation, used different ranges of frequencies for different moving windows of data.

A technique to improve CDP spatial resolution is needed for an effective exploration tool.

The temperature of the earth's surface is measured every day and night at various spatial resolutions from kilometers down to $90 \mathrm{~m}$ by a variety of remote sensing and POES weather satellites. Available 
spatial and temporal resolution is about to increase dramatically as proposed microsatellite systems from companies such as Planetary Resources become operational.

If a sufficiently long time series of temperatures is collected, common sense would suggest that the only signal constant over time would be heat flow from the mantle. Transient factors such as weather would be averaged out while thermal emittance from surficial geology could be modelled using day and night time temperature estimates.

\section{Heat flow from temperature times series}

We assume a simple linear model:

measured temperature $=$ surface reflection + surface emittance + atmospheric/weather related effects

+ slope/aspect/elevation effects + heat flow from the mantle

We are interested in estimating the last factor as an aid to kimberlite exploration as we'd like to flag areas with lithospheric conditions conducive to the production and preservation of carbon in diamond form, which requires relatively low heat flows of $40-45 \mathrm{~mW} \mathrm{~m}-2$ [3].

418 -day and 428 -night surface temperature averages collected by the MODIS satellite at $1 \mathrm{Km}$ spatial resolution during 2016 over the central Kalahari were downloaded from reverb.echo.nasa.gov. Last year was the peak of the worst drought in Southern Africa for a century so transient surface water effects are minimized. We also included a $1 \mathrm{Km}$ resolution DTM subsampled from Aster satellite stereo pairs from earthexplorer.usgs.gov to account for elevation effects.

Published heat flows for the ORZ [2] are used as the target variable in a multiple linear regression model and the temperature time series and DTM are explanatory variables. We are using magnetic derived heat flows to calibrate our temperature/DTM model. The estimates correlate at 0.74 .

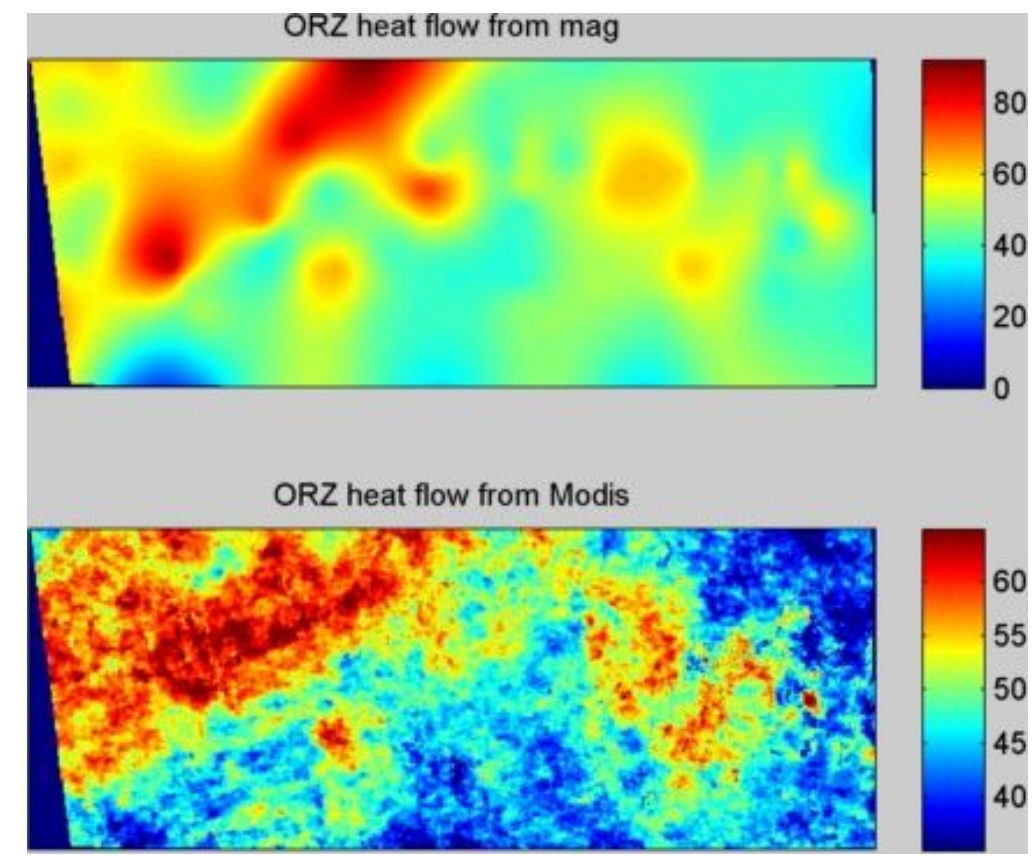

Figure 2: CDP from regional magnetics (top) and Modis temperatures (bottom)

The model is then extended to central Botswana for which no target CDPs are available. 


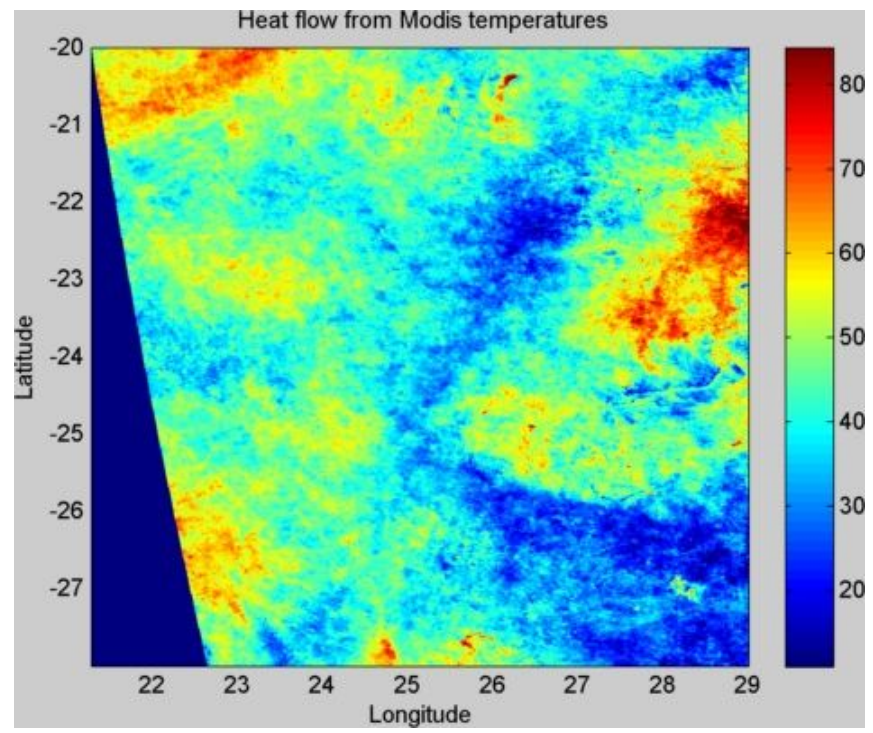

Figure 3: Modis heat flow for central Botswana

Visual inspection of kimberlite locations in the Jwaneng area confirms a spatial correlation with low heat flow regions.

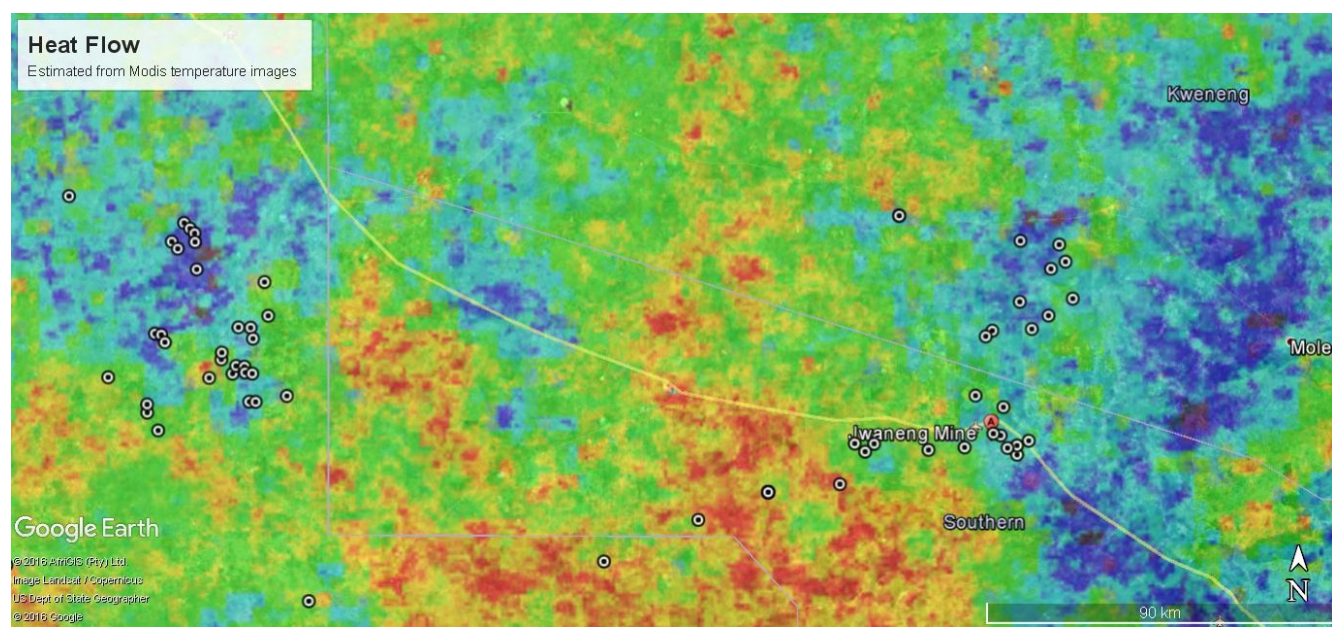

Figure 4: Modis heat flows for Jwaneng region

Heat flows can be used as an inexpensive reconnaissance tool in a regional exploration program.

\section{References}

1. Li, C.-F. et al. (2017). A global reference model of Curie-point depths based on EMAG2. Sci. Rep. 7, 45129; doi: 10.1038/srep45129.

2. Leseane, K., Atekwana, E. A., Mickus, K. L., Abdelsalam, M. G., Shemang, E. M. and Atekwana, E. A. (2015), Thermal perturbations beneath the incipient Okavango Rift Zone, northwest Botswana. J. Geophys. Res. Solid Earth, 120: 1210-1228. doi: 10.1002/2014JB011029.

3. Bouguern, A., Allek, K., Khalifa M., Bendiab, F. and D. Bouboya (2015), https://www.researchgate.net/publication/282161742_Mapping_curie_point_depth_of_the_w est_African_Craton_from_satellite_magnetic_data_and_its_implication_for_diamond_explor ation. 